\title{
Comparisons in prosomal width and body weight among early instar stages of Malaysian horseshoe crabs, Carcinoscorpius rotundicauda and Tachypleus gigas in the laboratory
}

\begin{abstract}
The three Southeast Asian horseshoe crab species are diminishing not only locally but also regionally and protection of them is now an urgent matter. Two species of horseshoe crab, Tachypleus gigas and Carcinoscorpius rotundicauda, were artificially inseminated, and the eggs were incubated at $28 \pm 1{ }^{\circ} \mathrm{C}$ and in the salinity of $33 \pm 2 \mathrm{ppt}$. The fertilized eggs hatched after 42 days and 41 days with hatching rates of 98.1 and $98.9 \%$ for $\mathrm{T}$. gigas and $\mathrm{C}$. rotundicauda, respectively. This study reveals that in the identical laboratory condition, $\mathrm{C}$. rotudicauda underwent more frequent molting than T. gigas. After 328 days of rearing, 63.8 and $22.9 \%$ of $\mathrm{C}$. rotundicauda larvae had molted to the 6 th and 7 th instars, respectively, while 56.6 and $20.1 \%$ of $\mathrm{T}$. gigas at the end of 355 days of rearing had molted to the 5 th and 6 th instars, respectively, but only $0.6 \%$ had molted to the 7 th instar. There is a wide variation in the molting rate among larvae obtained from synchronized inseminated eggs and reared under uniform laboratory conditions.
\end{abstract}

Keyword: Horseshoe crab; Inseminated 